\title{
Transit “Pass-Through" Lanes at Freeway Interchanges: A Life-Cycle Evaluation Methodology
}

\author{
Michael Mandelzys and Bruce Hellinga \\ University of Waterloo
}

\begin{abstract}
Transit "pass-through" lanes provide transit vehicle priority at freeway interchanges. "Pass-through" lanes allow a transit vehicle to exit the freeway at an interchange, cross straight through the intersecting arterial road, and re-enter the freeway. This treatment allows transit vehicles to bypass congestion on the mainline between the beginning of the off-ramp and the end of the on-ramp.

This paper outlines a methodology to evaluate if transit "pass-through" lanes are economically justified at a given interchange and provides a method for prioritizing candidate locations. The methodology provides an objective and consistent decision making method, reduces the effort required for practitioners to assess the need for "pass-through" treatment at a given interchange, and helps ensure that limited resources are directed towards interchanges that are expected to experience the greatest benefit per dollar spent.

The proposed methodology is based on an analytical approach that compares the value of travel time savings (for passengers and transit vehicles) with the construction and maintenance costs of the transit "pass-through" lane treatment.
\end{abstract}




\section{Introduction}

Transit vehicle priority is the preferred treatment of one vehicle class (transit) over other vehicle classes at a road network element (Smith et al. 2005). The provision of transit vehicle priority is often motivated by opportunities to reduce person-delay within the transportation network, increase transit reliability and speed, reduce transit operating costs, and/or encourage transit use due to the environmental and social benefits often associated with transit. Within a freeway environment, one potential form of transit priority is a transit "pass-through" lane (or bus bypass). "Pass-through" lanes allow a transit vehicle to exit the freeway at an interchange, cross the intersecting arterial road, and re-enter the freeway (Figure 1). This treatment allows transit vehicles to bypass congestion on the mainline between the beginning of the off-ramp and the end of the on-ramp. Transit "pass-through" lanes may use dedicated lanes and transit signal priority (TSP) at intersections to increase their effectiveness.

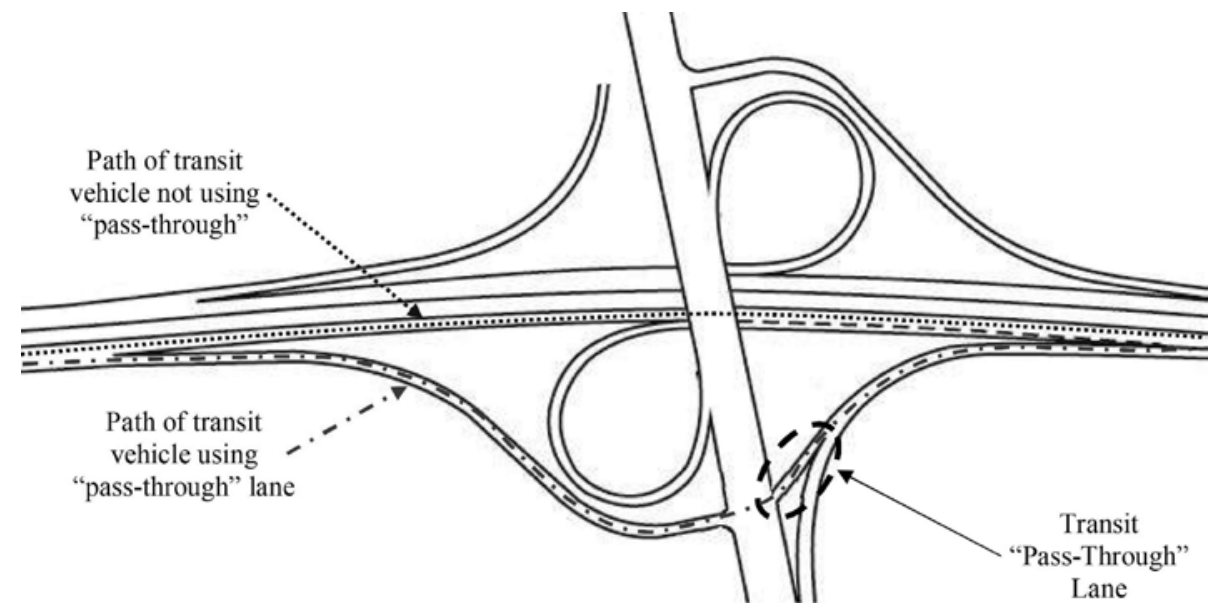

Figure 1. Transit "Pass-Through" Lane

In many situations, new transit "pass-through" lanes are implemented in conjunction with scheduled maintenance, rehabilitation, or construction of interchanges. However, there is a lack of a methodology, both in practice and in the literature, for evaluating whether a specific interchange is a worthwhile location for constructing a "pass-through" lane. Further, there is a benefit to being able to rank candidate interchanges such that locations with the greatest benefits are prioritized, allowing limited funds to be spent effectively. 
The evaluation and ranking of priority treatments can be done on the basis of relative benefits and costs associated with the treatments. In practice, detailed benefit/cost ranking tends to be cumbersome and time-consuming to conduct; therefore, it can be beneficial to embed the benefit/cost analysis within a simplified warrant procedure.

This paper outlines a warrant methodology that can be used to aid in determining whether or not construction of a transit "pass-through" lane at a given interchange is justified and provides a method for prioritizing candidate locations. The warrant methodology provides an objective and consistent decision making method, reduces the effort required for practitioners to assess the effectiveness of a "pass-through" treatment at a given interchange, and helps ensure that limited resources are directed towards interchanges that are expected to experience the greatest benefit per dollar spent.

The proposed methodology is based on an analytical approach to estimate expected daily travel time savings (for passengers and for transit vehicles) associated with providing transit "pass-through" lanes. The expected benefits of the treatment are derived by converting travel time savings into a dollar value. Costs of the treatment are estimated on the basis of annualized construction cost and estimated annual maintenance costs. The output of the methodology is a benefit/ cost ratio (BCR).

\section{Methodology}

Transit priority treatments are often evaluated via analytical or microsimulation methods. To provide the repeatability and ease of use typically associated with a warrant methodology, the procedure outlined in this paper is based on analytical methods.

The ultimate output of the warrant methodology is a BCR. If the BCR exceeds a certain threshold (typically 1.0), the proposed transit "pass-through" is evaluated as economically warranted. The BCR is also useful for comparing potential interchanges (FHWA 2003) and prioritizing those interchanges that will receive the greatest benefit per dollar spent.

The warrant methodology analyzes typical weekday conditions from 6:00 a.m. until 9:00 p.m., broken up into 15-minute periods to capture temporal variations in traffic conditions and bus frequencies. Data requirements to complete the warrant methodology consist of: 
- Freeway segment length $(\mathrm{km})$

- Bypass segment length $(\mathrm{km})$

- Freeway speed profile ( $\mathrm{km} / \mathrm{hr}$, per 15 -minute period)

- Off-ramp volume for lane group used for bypass (veh, per 15-minute period)

- Intersection configuration

- Heavy vehicle percentage for lane group used for bypass (\%)

- Traffic signal timing plan

- Transit signal priority parameters, if applicable

- Transit vehicle schedule

- Transit vehicle loadings (passengers/vehicle)

- Capital (construction) cost of bypass infrastructure (\$)

- Service life of bypass infrastructure (years)

- Annual maintenance cost of bypass infrastructure $(\$)$

\section{Benefit Estimation}

The benefit estimation portion of the warrant methodology involves estimating the travel time savings for transit vehicle passengers and the travel time savings for transit vehicles. These two values are used to quantify benefits such as reduced travel time for users, reduced vehicle requirements for transit agencies, reduced transit vehicle fuel consumption, and potential modal shifts from personal vehicles to transit among commuters.

The benefit estimation procedure is summarized in Figure 2 and consists of the following steps.

\section{Benefit Calculation Step 1: Construct Freeway Travel Time Profile}

Travel time for a bus along the mainline of the freeway (i.e., assuming the proposed transit "pass-through" lane is not used) is estimated for each 15-minute time period throughout the day. Travel time is calculated for each period based on freeway speeds (typically measured using loop detectors or other dedicated traffic sensors) in the vicinity of the interchange and the distance along the mainline that could be skipped by using the bypass (Equation 1). 


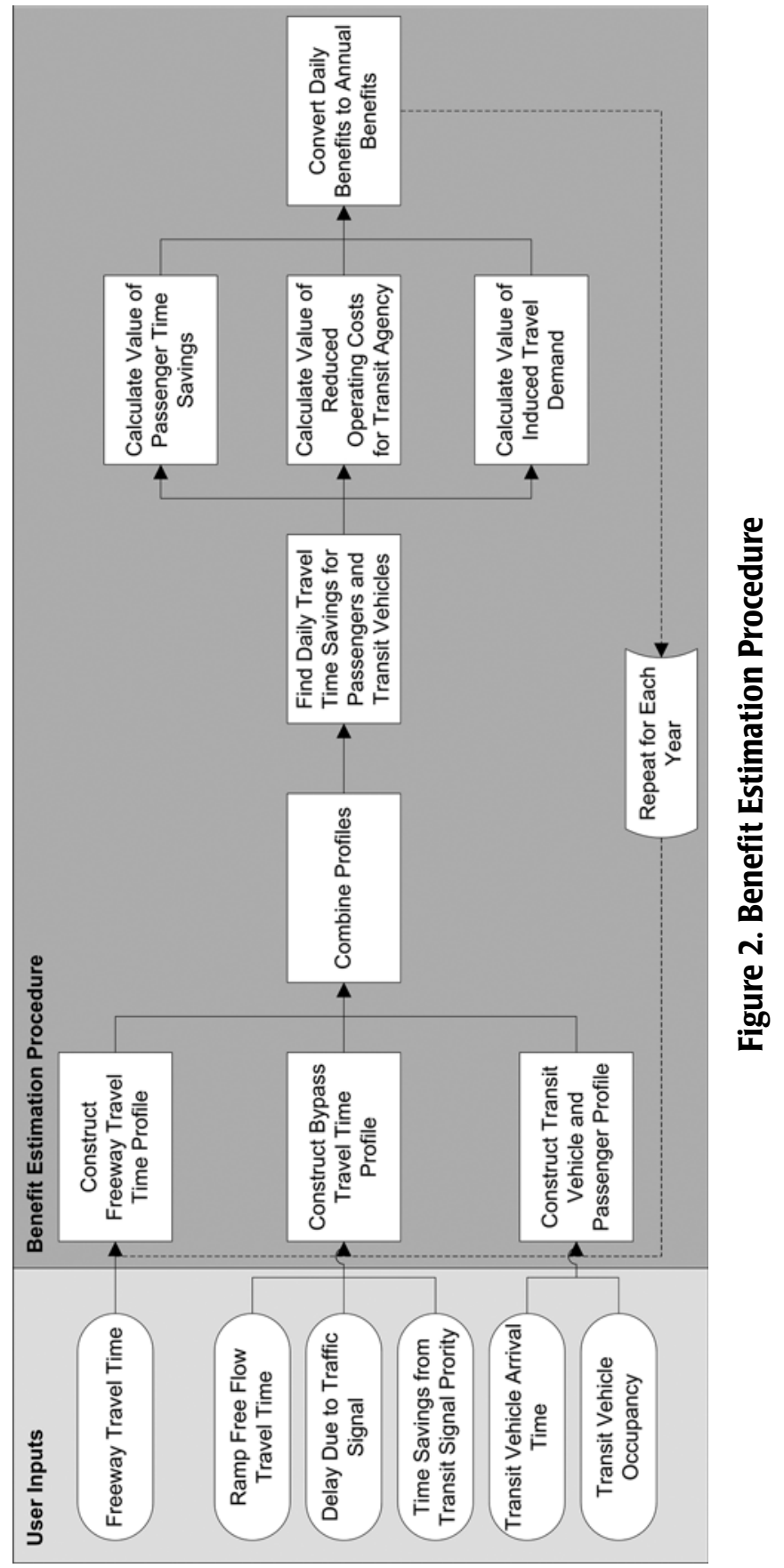


$T T_{\text {Freeway }, i}=\frac{3600 \cdot D_{\text {Freeway }}}{V_{\text {Freeway }, i}}$

Where $T T_{\text {Freway, } i}$ is the travel time on the freeway in period $i$, in seconds

$D_{\text {Freway }}$ is the distance along the freeway which the transit vehicle would avoid if it used the bypass, in $\mathrm{km}$

$V_{\text {Freway, } i}$ is the speed on the mainline freeway in period $i$, in $\mathrm{km} / \mathrm{hr}$

The resulting output of this step is a freeway travel time profile over the course of a typical weekday. It is also possible to construct the freeway travel time profile directly using observed/archived travel time data for the freeway in the vicinity of the interchange.

\section{Benefit Calculation Step 2: Construct Bypass Travel Time Profile}

Travel time for a bus using the transit "pass-through" lane is based on free-flow travel time along the bypass route, plus an additional delay due to the traffic signal at the arterial road crossing, minus some time savings from TSP if it is provided. Conceptually, the travel time for the bypass is calculated during each period as follows (Equation 2):

$T T_{\text {Bypass }, i}=T T_{\text {BypassFreeflow }}+T T_{\text {Signal,i }}-T T_{T S P, i}$

Where $T T_{\text {Bypass,i }}$ is the travel time on the bypass in period $i$, in seconds

$T T_{\text {Bypassfreeflow }}$ is the travel time on the bypass assuming free-flow conditions, in seconds

$T T_{\text {signal, } i}$ is the additional travel time added by the traffic signal at the crossing arterial road during period $i$, in seconds

$T T_{T S P, i}$ is the travel time savings attributable to transit signal priority at the traffic signal at the crossing arterial road during period $i$, in seconds

The travel time for the bypass under free-flow conditions is an idealized time that assumes that the route could be completed without the need to stop or slow 
due to the traffic signal or queues at the traffic signal. This free-flow travel time is, therefore, limited by the geometry and speed limit of the bypass route. Calculation of travel time for the bypass under free-flow conditions is indicated in Equation 3. Since this value is independent of traffic volumes and signal operation, it is constant during all time periods.

$T T_{\text {BypassFreeflow }}=\frac{3600 \cdot D_{\text {Bypass }}}{V_{\text {Bypass Freflow }}}$

Where $D_{\text {Bypass }}$ is the distance travelled on the bypass, in $\mathrm{km}$

$V_{\text {Bypassfreeflow }}$ is the average free-flow speed on the bypass, in $\mathrm{km} / \mathrm{hr}$

Having to cross an arterial road at a traffic signal adds travel time to the bypass. The amount of additional travel time is a function of traffic volumes, signal timings, driver behavior, and intersection configuration and will therefore vary throughout the day. The additional delay due to the traffic signal during each period is estimated by following the methodology outlined in Chapter 16 of the Highway Capacity Manual 2000 (Transportation Research Board 2000), as outlined in Equation 4.

$T T_{\text {Signal, } i}=d_{1}+d_{2}+d_{3}$

Where $d_{1}$ is the uniform control delay based uniform arrivals, in seconds

$d_{2}$ is the incremental delay due to random arrivals and oversaturation queues, in seconds

$d_{3}$ is the initial queue delay, in seconds

The delay due to the traffic signal can be partially mitigated through the provision of transit signal priority. To quantify the expected delay reduction due to transit signal priority, a simplified analytical model has been used (Lin 2002). The model presents expected delay reduction as a function of the "aggressiveness" of the transit signal priority parameters, i.e., the maximum green extension and red truncation permitted (Equation 5). 
$T T_{T S P, i}=\frac{\delta}{C} R+\frac{R^{2}-R_{\min }^{2}}{2 C}$

Where $C$ is the cycle length, in seconds

$R$ is the length of red phase for the bus approach, in seconds

$R_{\text {min }}$ is the minimum permissible red phase for the bus approach, in seconds

$\square$ is the maximum permissible green extension for the bus approach, in seconds

Note that the total signal delay $\left(T T_{\text {Signal,i }}\right)$ acts as an upper bound on the travel time savings due to TSP $\left(T T_{T S P, i}\right)$.

The resulting output of this step is a bypass travel time profile over the course of a typical weekday.

\section{Benefit Calculation Step 3: Construct Transit Vehicle and Passenger Profile}

A daily profile of transit use (in terms of both number of passengers and number of vehicles) must be known to evaluate the effectiveness of a proposed bypass. The profile can be created based on a known or planned transit schedule and based on a known or assumed bus occupancy level. The profile must identify the number of buses and passengers expected during each period

\section{Benefit Calculation Step 4: Combine Profiles and Find Daily Travel Time Savings}

The daily travel time savings, in terms of passenger hours and transit vehicle hours saved, can be found. by combining the profiles created in steps 1 to 3 .

The transit "pass-through" lane provides a benefit only during periods in which a transit vehicle's travel time using the bypass is less than its travel time using the freeway. During periods when this is not the case, it is likely that the transit vehicle will simply stay on the freeway, and the bypass will not be used. As well, regardless of the difference in travel times between the freeway and the bypass, travel time savings can be accrued only during periods in which transit vehicles are scheduled to arrive. Therefore, travel time savings exist only during specific periods of the day. Travel time savings during each of these periods can be calculated as the difference between travel time on the bypass and travel time on the freeway multiplied by 
either the number of passengers or the number of vehicles. Total daily travel time savings will be the sum of these values over the course of the day, as indicated in Equations 6 and 7 .

$\Delta T T_{\text {Pass }}=\frac{1}{3600} \sum_{i=1}^{n}\left(\left\{\begin{array}{cl}T T_{\text {Bypass }, i}-T T_{\text {Freeway, } i} ; T T_{\text {Bypass }, i}<T T_{\text {Freeway }, i} \\ 0 \quad ; T T_{\text {Bypass }, i} \geq T T_{\text {Freeway }, i}\end{array}\right) \cdot N_{\text {Passenger }, i}\right.$

Where $\Delta T T_{\text {Pass }}$ is the daily passenger travel time savings due to the bypass, in hours

$T T_{\text {Bypass,i }}$ is the travel time on the bypass during period $i$, in seconds

$T T_{\text {Freeway, } i}$ is the travel time on the freeway during period $i$, in seconds

$N_{\text {Passenger, } i}$ is the number of passengers on the transit vehicles in period $i$

$n \quad$ is the number of 15 -minute periods from 6 a.m. to 9 p.m. $(n=60)$

$\Delta T T_{\text {Bus }}=\frac{1}{3600} \sum_{i=1}^{n}\left(\begin{array}{cl}T T_{\text {Bypass }, i}-T T_{\text {Freeway, } i} ; T T_{\text {Bypass }, i}<T T_{\text {Freeway }, i} \\ 0 \quad ; T T_{\text {Bypass }, i} \geq T T_{\text {Freeway }, i}\end{array}\right) \cdot N_{\text {Bus }, i}$

Where $\Delta T T_{\text {Bus }}$ is the daily transit vehicle travel time savings due to the bypass, in hours

$T T_{\text {Bypass,i }}$ is the travel time on the bypass during period $i$, in seconds

$T T_{\text {Freeway, } i}$ is the travel time on the freeway during period $i$, in seconds

$N_{\text {Bus,i }} \quad$ is the number of transit vehicles in period $i$

\section{Benefit Calculation Step 5: Convert Daily Travel Time Savings into Annual Dollar Value Benefits}

The additional passenger travel time savings and transit vehicle travel time savings have several benefits that are considered in this warrant methodology. There is the inherent value of passenger time that is saved due to the provision of the bus "pass-through" lane. The U.S. Department of Transportation recommends a value of time equal to average wage plus value of fringe benefits for business travel and 
$50 \%$ of average wage for personal travel (Kruesi 1997, Frankel 2003). According to the National Compensation Survey published by the U.S. Department of Labor (2007), earnings in the United States averaged $\$ 19.29$ /hour, so a value of passenger time of about $\$ 15 /$ person-hour may be a reasonable starting point and can been selected by default. Practitioners may modify this value from the default based on their own experience of local conditions and values.

Travel time savings also benefit transit service agencies, since they can result in reduced bus operating times and a corresponding reduction in agency operating costs. To get a significant benefit, time savings should be high enough to reduce the number of transit vehicles the agency needs to operate a route. However, this can be difficult to quantify, since one individual transit "pass-through" lane at an interchange may not provide sufficient time savings on its own, but could be sufficient in combination with other improvements such as "pass-through" lanes at other interchanges, TSP, transit schedule changes, and more. By default, a value of $\$ 80 /$ bus-hour may be used to represent the value of transit vehicle time savings to the transit agency. This value can be modified based on the experience of the affected transit agencies. The default value has been calculated based by dividing total 2007 bus operating expenses by total 2007 bus operating hours for transit systems across the United States (National Transit Database) and provides an approximation of the cost to run transit services on a per-hour operated basis.

A third benefit is that by improving the performance of transit, it becomes more attractive relative to auto use. This has the potential to induce transit demand. The shift of travelers from personal vehicles to transit has obvious benefits such as a decrease in the number of vehicles on the road (reduced congestion), reduced emissions, etc. It is difficult to quantify the level and value of induced transit demand attributable to the reduction in travel time on a transit route. By default, the warrant methodology uses a value of $\$ 0 /$ person-hour for this benefit, which means it is not accounted for in the warrant. However, an agency may wish to modify this value based on its experience or in-house data that supports a higher value.

Total daily benefits can be found by multiplying the daily travel time savings by the appropriate conversion factors (Equation 8):

$B_{\text {Daily }}=\Delta T T_{\text {Pass }} \cdot \alpha_{\text {Time }}+\Delta T T_{\text {Pass }}+\alpha_{\text {OpCost }}+\Delta T T_{\text {Bus }} \cdot \alpha_{\text {InducedDemand }}$ 
Where $B_{\text {Daily }} \quad$ is the daily value of the benefits, in $\$$

$\alpha_{\text {Time }}$ is the passenger car value of time, in $\$$ /passenger-hour

$\alpha_{\text {opcost }}$ is the value of reduced bus operating times, in $\$ /$ passenger-hour

$\alpha_{\text {InducedDemand }} \quad$ is the value of induced transit demand, in $\$ /$ bus-hour

Next, the daily benefits are converted into annual benefits by multiplying by the number of weekdays with transit service in a year (Equation 9).

$B_{j}=B_{\text {Daily }} \cdot$ ServiceWeekdays

Where $B_{j} \quad$ is the annual value of the benefits during year $j$, in $\$$

ServiceWeekdays is the number of weekdays per year on which a transit service operates, in days

\section{Benefit Calculation Step 6: Repeat Calculations for each Year to Find Benefit Annuity}

Equation 9 yields the total value of benefits accrued during the analyzed year (year j). Since conditions are likely to change from year to year (such as increased travel times on the mainline freeway or increased transit service/ridership), $B_{j}$ can be recalculated for each year over the service life of the transit "pass-through" lane. The benefits calculated for each year are then brought back to time zero, summed, and converted to an annuity over the entire service life of the transit "pass-through" lane (Equation 10).

$B=\left(\sum_{j=0}^{n-1} \frac{B_{j}}{(1+i)^{(j+1)}}\right) \cdot \frac{i(i+1)^{n}}{(1+i)^{n}-1}$

Where $B \quad$ is the benefit annuity, in \$

$i \quad$ is the annual interest rate used by the agency to represent the time-value of money

$n \quad$ is the service life of the infrastructure, in years 
Evidently, this step significantly increases the data and workload requirements of the warrant procedure, since the calculation of $B_{j}$ for each year requires recomputing all the previous steps for each year.

In many situations, the methodology can be simplified by assuming that conditions remain constant over the service life of the improvement. Although this assumption is not strictly true, it greatly simplifies the calculation of the benefit annuity, $B$, such that it is simply equal to the annual benefits calculated for year $0\left(B_{0}\right)$. The assumption will frequently result in a conservative bias in the warrant methodology since, in most cases, conditions in the future tend to favor improvements more so than conditions today. This is because congestion is frequently projected to increase and, correspondingly, traffic speeds on the freeway are being reduced as time goes on. Further, transit service/usage is typically expected to remain constant or increase at locations where transit improvements are being considered. Both these factors have the potential to lead to even greater benefits from a transit "pass-through" lane in future years. By not accounting for these factors, we are frequently providing a conservative benefit of the true estimates. Therefore, when using this simplifying assumption, if a "pass-through" is warranted using the current methodology, then it would likely also be warranted had speed profile changes over time been taken into account.

In general, it is recommended that the warrant be completed first with the simplifying assumption that conditions remain constant. Unless freeway speeds are expected to increase in the future, or transit use is expected to decrease, an interchange that meets the warrant requirements with this simplifying assumption should also meet the warrant requirements if changes in conditions had been accounted for. In situations where significant changes in travel time or transit profiles are expected over the service life of the transit "pass-through" lane, it may be worthwhile to discard the simplifying assumption and calculate the benefits for each year as outlined in this step to determine if the results of the warrant are significantly affected.

\section{Cost Estimation}

Costs of a transit "pass-through" lane treatment are estimated on the basis of construction and maintenance costs. The cost estimation procedure is summarized in Figure 3 and consists of the following steps. 


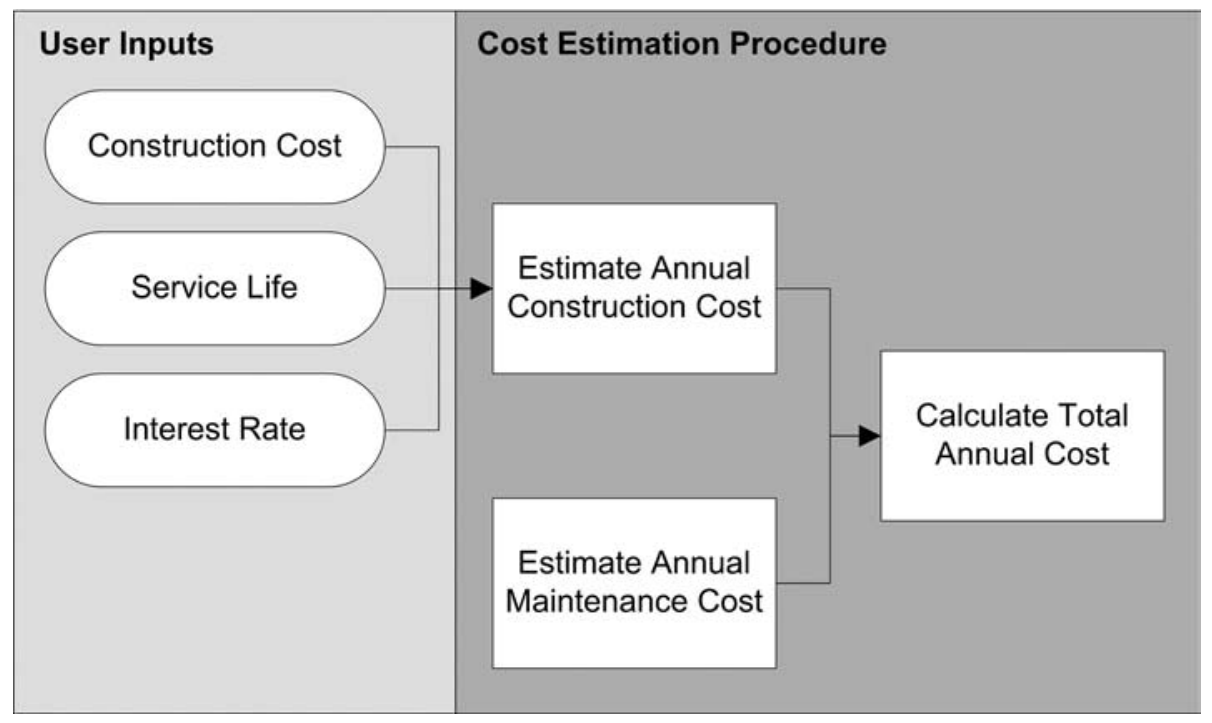

Figure 3. Cost Estimation Procedure

\section{Cost Calculation Step 1: Estimate Annual Construction Cost and Annual Maintenance Cost}

Once the construction cost is estimated, it can be converted in to an annual value over the service life of the infrastructure using Equation 11.

$A \mid C_{\text {Construction }}=C_{\text {Construction }} \cdot \frac{i(1+i)^{n}}{(1+i)^{n}-1}$

Where $A \mid C_{\text {construction }}$ is the annual value of the construction cost, in $\$$

$$
C_{\text {construction }} \quad \text { is the construction cost, in } \$
$$

The maintenance cost should be expressed as an annual cost over the service life of the infrastructure.

\section{Cost Calculation Step 2: Calculate Total Annual Cost}

The total cost of a proposed transit "pass-through" lane is the sum of the annualized construction cost and the maintenance costs (Equation 12). 


$$
C=A\left|C_{\text {Construction }}+A\right| C_{\text {Maintenance }}
$$

Where $C$

$$
A \mid C_{\text {Maintenance }} \quad \text { is the annual maintenance costs, in } \$
$$

The full warrant methodology has been implemented in an automated spreadsheet format to ease its application.

\section{Interpretation}

The ultimate output of the warrant is a benefit/cost ratio (BCR). The transit "passthrough" lane meets the minimum requirements of the warrant when the BCR exceeds a certain threshold. Typically, this threshold will be 1.0 (benefits exceed costs); however, individual agencies should have some flexibility in the threshold for meeting the warrant. This flexibility recognizes that the warrant represents a simplified BCR and that its results are subject to the assumptions and limitations as outlined previously.

In addition to evaluating whether a transit "pass-through" lane is warranted at a given location, the warrant methodology can be used to easily compare multiple potential locations. Locations that meet the minimum requirements of the warrant can be ranked from highest $B C R$ to lowest $B C R$, which allows those locations that are expected to experience the greatest benefit per dollar spent to be prioritized over locations that also meet the minimum warrant requirements but provide relatively lower benefits for the investment.

\section{Assumptions and Limitations}

When developing a warrant methodology, there is a need to find an appropriate balance between complexity and accuracy. The time and data requirements to complete the warrant methodology should not act as an impediment to its use, while still ensuring that the output of the warrant is of sufficient accuracy to allow the warrant be used as the decision making tool it is intended to be.

To achieve this balance, the proposed warrant methodology relies on several assumptions to simplify application and minimize excessive data requirements. The following key assumptions are made in this warrant methodology:

- HCM 2000 signalized delay calculations are applicable. Since this warrant methodology uses the HCM 2000 signalized delay equations to estimate the 
delay experienced by the transit vehicle when passing through the signalized intersection, the assumptions included in the HCM 2000 method are inherently part of this warrant methodology.

- Simplified TSP delay reduction equation is applicable. This methodology uses a simplified analytical equation to estimate expected delay reductions from transit signal priority. This equation makes several simplifying assumptions, including that the bus is detected and reacted to instantly by the TSP system and that buses have sufficient headways such that TSP system recovery time is not a factor (Lin 2002).

In addition to the assumptions discussed above, there are also several factors which are not considered in the warrant in order to maintain simplicity. These factors include the following and are discussed in greater detail by Mandelzys and Hellinga (2009):

- Disbenefit to cross-street traffic

- Improvements in service reliability

- Potential for transit stops at interchanges

\section{Application}

The application of the warrant methodology is illustrated for a freeway interchange (Highway 401 Eastbound/Avenue Road) in southern Ontario. This interchange had a transit "pass-through" lane constructed in 2007; however, the "passthrough" lane is not yet in use.

Highway 401 is a major freeway within the city of Toronto. The eastbound direction of Highway 401 operates with an express-collector configuration at Avenue Road, with the Avenue Road exit available only from the collector lanes. A full-day freeway speed profile was not available at this location; therefore, the freeway speed profile was estimated based on data collected in a 2006 travel time study for the Ontario Ministry of Transportation (MTO). The travel time study used probe vehicles and focused on peak AM, midday, and PM periods. Since there was a limited sampling frequency, travel times were interpolated during peak periods, and the freeway was assumed to be free-flowing at all other times. As well, to simplify calculations and because there was no information available to estimate future freeway travel time profiles or transit schedules/ridership, we have made the simplifying assumption that conditions remain constant in future years. The data sources used are summarized in Table 1. 


\section{Table 1. Highway 401 EB/Avenue Road Warrant-Data Sources}

\begin{tabular}{ll} 
Data & Source \\
\hline Freeway segment length & Measured from aerial photographs \\
Bypass segment length & Measured from aerial photographs \\
Freeway speed profile & 2006 Travel Time Study \\
Off-ramp lane group volume & MTO turning movement counts \\
Intersection configuration & MTO drawings \\
Heavy vehicle percentage & MTO turning movement counts \\
Traffic signal timing plan & City of Toronto \\
TSP parameters & n/a \\
Transit vehicle schedule & Existing transit schedules \\
Transit vehicle loadings & Full buses assumed $(52$ passengers $)$ \\
Construction cost & Discussions with MTO $(\$ 500,000)$ \\
Service life & Discussions with MTO $(30$ years $)$ \\
Maintenance cost & Discussions with MTO $(\$ 10,000 /$ year $)$
\end{tabular}

a Data available only for a portion of the study period; travel speeds were interpolated during peak periods and assumed to be free-flowing at other times.

The travel time and transit profiles found by applying the warrant methodology are illustrated in Figure 4. Based on the profiles, the transit "pass-through" lane would provide a significant time savings during the PM peak period and a moderate time savings during small portions of the $A M$ and midday peak period. During the rest of the day, no benefits are expected to be accrued from the transit "passthrough" lane because freeway speeds are fast enough that transit vehicles would not be using the "pass-through" lane.

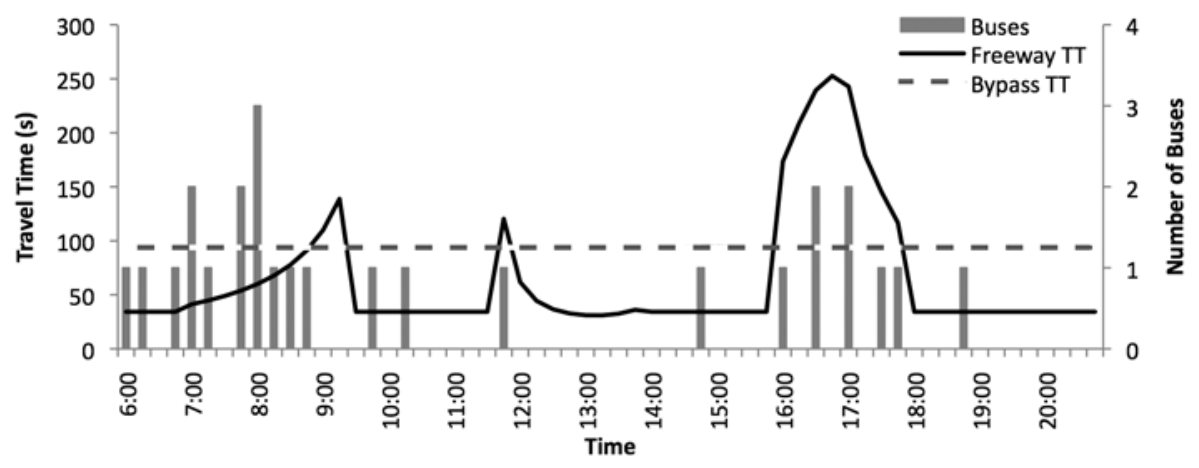

Figure 4. Highway 401 EB/Avenue Road Travel Time and Transit Profile 
Based on the profiles constructed using the warrant methodology, the final warrant calculations are summarized in Table 2. Ontario-specific values to convert travel time savings to dollar benefits ( $\$ 15 /$ passenger-hour for passenger time savings, $\$ 90 /$ bus-hour for reduced agency operating costs) were used in the final calculations.

\section{Table 2. Highway 401 EB/Avenue Road Warrant-Results}

\begin{tabular}{ll} 
Item & Value \\
\hline Daily Passenger Travel Time Savings (person-hours) & 11.1 \\
Daily Bus Travel Time Savings (bus-hours) & 0.21 \\
Daily Benefits (\$) & 185.99 \\
Annual Benefits (\$) & $46,498.51$ \\
Construction Cost (\$) & $500,000.00$ \\
Annualized Construction Cost (\$) & $32,525.72$ \\
Annualized Maintenance Cost (\$) & $10,000.00$ \\
Total Cost (\$) & $42,525.72$ \\
BCR & 1.093 \\
\hline
\end{tabular}

The results of the warrant analysis indicate that benefits are expected to exceed costs for a transit "pass-through" lane at this interchange. It had been assumed that there were no changes in the travel time or transit profiles over the service life of this improvement. In reality, there is likely to be increased congestion in the future and the same or more frequent transit service, although no data are available to quantify the magnitude of this change. These changes would lead to increased benefits; however, the recommendation of the warrant should not be affected (the transit "pass-through" lane would still be economically justified at this location). Ultimately, the final BCR can be compared with warrant results at other locations to prioritize candidate locations.

\section{Discussion}

Previous sensitivity analysis by Mandelzys and Hellinga (2009) of the input parameters had found that freeway travel time plays a significant factor in the outcome of the warrant analysis. If the freeway does not experience significant congestion during periods when transit vehicles use the freeway, the warrant is unlikely to be met. Conversely, high levels of freeway congestion significantly increase the benefits of a transit "pass-through" lane. The effect of ramp volume on warrant 
outcome was found to be minimal, except when ramp volumes approached or exceeded capacity. The transit schedule was found to be important, since benefits can be accrued only during time periods when transit vehicles actually travel through the study area. Finally, the choice of multiplication factors (to convert time savings to benefits) was found to affect the BCR in a linear manner with the rate of change being proportional to the amount of time savings expected

In consideration of these findings, Mandelzys and Hellinga (2009) recommended that full data collection is unlikely to be needed for the entire 6 a.m. to 9 p.m. period. Instead, with minimal impact on the output of the warrant methodology, data collection could be limited to periods containing any one of:

- notable freeway congestion

- high ramp volumes

- notable transit volumes

Under most circumstances, the time periods of the above three cases can be expected to roughly coincide.

\section{Conclusions}

One form of providing transit vehicle priority within a freeway environment is to create transit "pass-through" lanes at interchanges. "Pass-through" lanes allow a vehicle to exit the mainline of the freeway at an off-ramp, cross straight across the intersecting arterial road, and re-enter the freeway via the on-ramp. When the mainline of the freeway is heavily congested, this allows the transit vehicle to bypass a significant portion of the freeway.

These treatments frequently are implemented on an ad-hoc basis, and there is a lack of a consistent methodology to determine if the benefits of implementing a transit "pass-through" lane treatment at a given location justify the associated costs. This paper outlines a warrant methodology that can be used to test individual candidate interchanges and to rank the locations such that interchanges with the greatest relative benefits are prioritized over interchanges with lower relative benefits. The output of the warrant methodology is a benefit/cost ratio.

It was found that freeway speeds have a significant influence on the results of the warrant analysis. If freeway speeds are generally high throughout the day, the warrant is unlikely to be met. Lane group volumes at the signalized intersection of the off-ramp have a smaller effect on the outcome of the warrant, unless volumes 
approach or exceed capacity. The transit schedule is also important, as travel time benefits are accrued only during periods in which transit vehicles pass through the interchange. Therefore, the key periods for the warrant to analyze should include times when (a) there is significant freeway congestion, (b) there are highvolumes on the transit "pass-through" lane group, or (c) there are notable transit volumes.

This methodology forms a good basis for analyzing potential interchanges for transit "pass-through" lanes in the future. The methodology is beneficial as it provides an objective and consistent decision making method, reduces the effort required to assess the need for "pass-through" treatment at a given interchange, and ensures that limited resources are directed towards interchanges that are expected to experience the greatest benefit per dollar spent.

\section{Recommendations}

A transit "pass-through" lane treatment would seem to interact well with bus-onshoulder operations, since it can eliminate the need to exit the shoulder and cross over mixed traffic at the interchanges. The precise benefits may vary by application, but they are not currently accounted for in the methodology. Accounting for the benefit of combining transit "pass-through" lanes with bus-on-shoulder operations may be an area for future research.

This methodology is limited to estimating effects at a single interchange. Ultimately, it would be beneficial for the methodology to include a mechanism for considering an entire corridor of interchanges, since this would allow interactions between interchanges and the cumulative effects of time savings to be investigated more thoroughly. The analysis has been limited to single interchanges at this time to reflect the limited scale of implementation being considered by many transit agencies. It is our understanding that transit "pass-through" lane treatments often are considered for only a few interchanges and/or in conjunction with already scheduled interchange construction/maintenance/rehabilitation. A corridor-wide warrant methodology is a potential area for future research.

This methodology could be considered for inclusion in the Highway Capacity Manual or the Transit Capacity and Quality of Service Manual to disseminate the techniques to practitioners. 


\section{Acknowledgments}

The authors would like to thank the Ontario Ministry of Transportation for its technical support and feedback, as well as for funding this research. Special thanks is given to Ms. Nancy Adriano for her time and input in completing this research. The findings described in this paper are those of the authors and should not be interpreted to represent Ontario Ministry of Transportation policy.

\section{References}

FHWA. 2003. Economic Analysis Primer. Publication FHWA-IF-03-032. Washington, D.C.: Office of Asset Management, FHWA, U.S. Department of Transportation.

Frankel, E. H. 2003. Revised Departmental Guidance: Valuation of Travel Time in Economic Analysis. Office of the Secretary of Transportation, U.S. Department of Transportation.

Kruesi, F. E. 1997. Departmental Guidance for the Valuation of Travel Time in Economic Analysis. Office of the Secretary of Transportation, U.S. Department of Transportation.

Lin, W-H. 2002. Quantifying delay reduction to buses with signal priority treatment in mixed-mode operation. Transportation Research Record 1811: 100-106.

Mandelzys, M., and B. Hellinga. 2009. Warrant methodology for evaluating and ranking transit "pass-through" lanes at freeway interchanges. Paper presented at the annual meeting of the Transportation Research Board, January 11-15, Washington D.C.

National Transit Database. National Transit Database-Data-TS2.1 - Service Data and Operating Expenses Time-Series by Mode. http://www.ntdprogram. gov/ntdprogram/data.htm.

Smith, H. R., B. Hemily, and M. Ivanovic. 2005. Transit Signal Priority: A Planning and Implentation Handbook. ITS America.

Transportation Research Board. 2000. Highway Capacity Manual 2000. Washington, D.C.: Transportation Research Board. 
U.S. Department of Labor, U.S. Bureau of Labor Statistics. 2007. National Compensation Survey: Occupational Wages in the United States, June 2006. Summary 07-03. Washington, D.C.: U.S. Department of Labor.

\section{About the Authors}

MiChAel MANDELzYS (mike.civ@gmail.com) received an undergraduate degree in Civil Engineering from the University of Waterloo, followed by several years of work experience in transportation planning and design at LEA Consulting, a transportation consultant in Toronto. In 2007, he returned to the University of Waterloo as a graduate student and currently is pursuing an MASc with a research focus in transit.

Dr. BrUCe HeLlinga (bhellinga@uwaterloo.ca) received BASc and MASc degrees in Civil Engineering from the University of Waterloo in 1989 and 1990 and a PhD in Civil Engineering-Transportation from Queen's University in Kingston, Ontario, in 1994. Currently, he is an Associate Professor in the Department of Civil and Environmental Engineering at the University of Waterloo. He has authored or coauthored more than 90 technical papers and reports reflecting his research interests in traffic engineering and control, traffic and transit modeling, safety and ITS, with financial support from a number of public and private sector agencies including the Natural Sciences and Engineering Research Council of Canada and federal, provincial, and municipal transportation agencies. 